intensive care unit admission, elective or emergency operations and endoscopy. The COVID-19 period was defined as the time after the first local case in January 2020, week 4.

Results We recorded a total of 195,867 hospital admissions related to GI diseases during the study period, and 125,049 of them were included in the final analysis. Comparing the same pre-COVID19 and COVID-19 periods, we observed a significant decline in the average number of hospitalizations for GI diseases $(17.0 \%$ reduction, $P<0.001)$. (Figure 1) Inhospital mortality did not differ significantly $(11.2 \%$ vs $11.7 \%, P=0.063)$. Overall ICU admission rate was static $(5.3 \%$ vs $5.1 \%, P=0.218)$. More emergency endoscopies were performed in patients with UGIB $(30.0 \%$ vs $38.9 \%, P$ $<0.001)$ and benign pancreaticobiliary conditions $(17.5 \%$ vs $24.4 \%, P<0.001)$. Higher proportion of patients underwent emergency operations during their hospitalization $(6.7 \%$ vs $8.0 \%, P<0.001)$.

Conclusions The number of hospitalizations related to GI diseases reduced drastically during the COVID-19 epidemic, yet no excessive in-hospital mortality was observed. More emergency endoscopies and operations were required, particularly for UGIB and benign pancreaticobiliary conditions.

\section{IDDF2020-ABS-0140 WORLDWIDE INCIDENCE AND RISK FACTORS OF OESOPHAGEAL CANCER BY HISTOLOGICAL SUBTYPES}

${ }^{1} J u n j i e$ Huang*, ${ }^{1}$ Veeleah Lok, ${ }^{1}$ Cedric Chu, ${ }^{1}$ Chun Ho Ngai, ${ }^{2}$ Ping Chen, ${ }^{3}$ Shanjuan Wang, ${ }^{4}$ Lin Zhang, ${ }^{5}$ Jinqiu Yuan, ${ }^{1}$ Xiang-Qian Lao, ${ }^{1}$ Shelly LA Tse, ${ }^{6}$ Wanghong Xu, ${ }^{7}$ Zhi--jie Zheng, ${ }^{8}$ Shao-Hua Xie, "Martin Wong. 'Jockey Club School of Public Health and Primary Care, Faculty of Medicine, Chinese University of Hong Kong, Hong Kong; ${ }^{2}$ Department of Gastroenterology, Ruijing Hospital North, School of Medicine, Shanghai Jiaotong University, China; ${ }^{3}$ Department of Gastroenterology, Jiading District Hospital, China; ${ }^{4}$ Melbourne School of Population and Global Health, The University of Melbourne, Australia; ${ }^{5}$ Clinical Research Centre; Scientific Research Centre, The Seventh Affiliated Hospital, Sun Yat-sen University, China; ${ }^{6}$ School of Public Health, Fudan University, China; 'Department of Global Health, School of Public Health, Peking University, China; ${ }^{8}$ Upper Gastrointestinal Surgery, Department of Molecular Medicine and Surgery, Karolinska Institute, Karolinska University Hospital, Sweden

\subsection{6/gutjnl-2020-IDDF.86}

Background Worldwide, oesophageal cancer is one of the most common cancers and a leading cause of cancer mortality. Owing to its aggressive disease nature and poor survival rate, it contributes to a substantial burden to global health and clinical practice. This study aimed to estimate the worldwide

(Males)

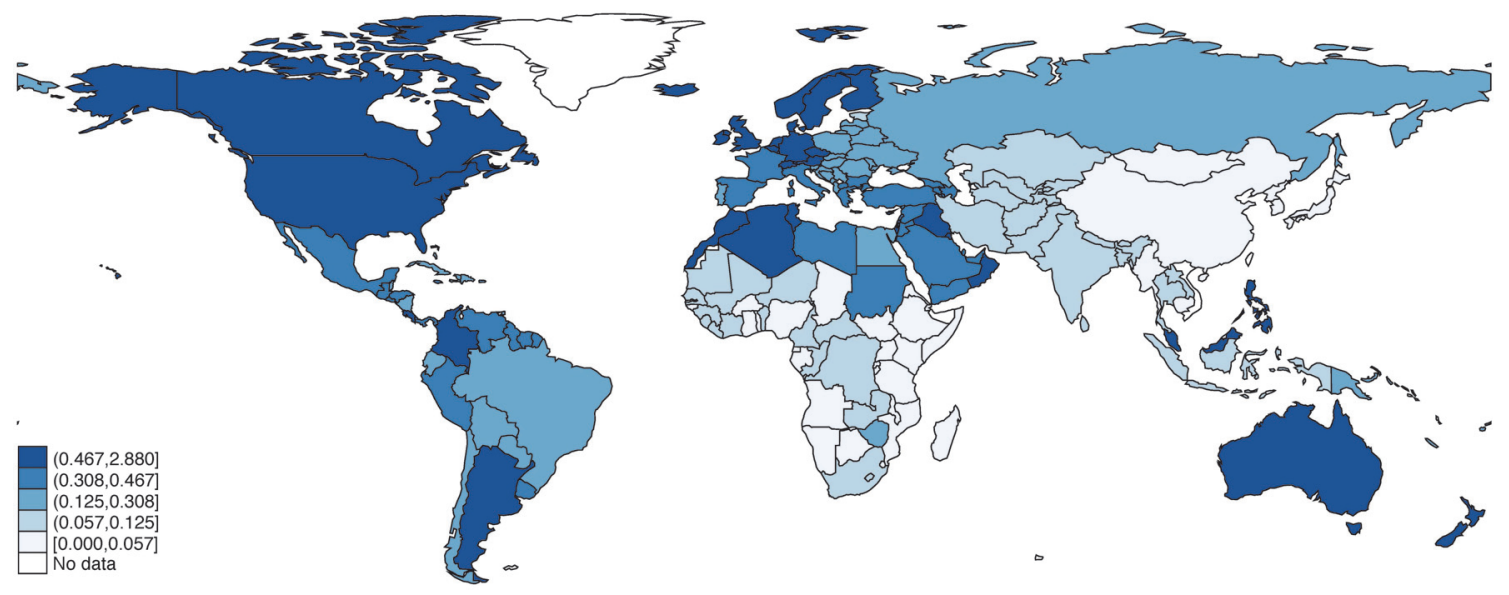

(Females)
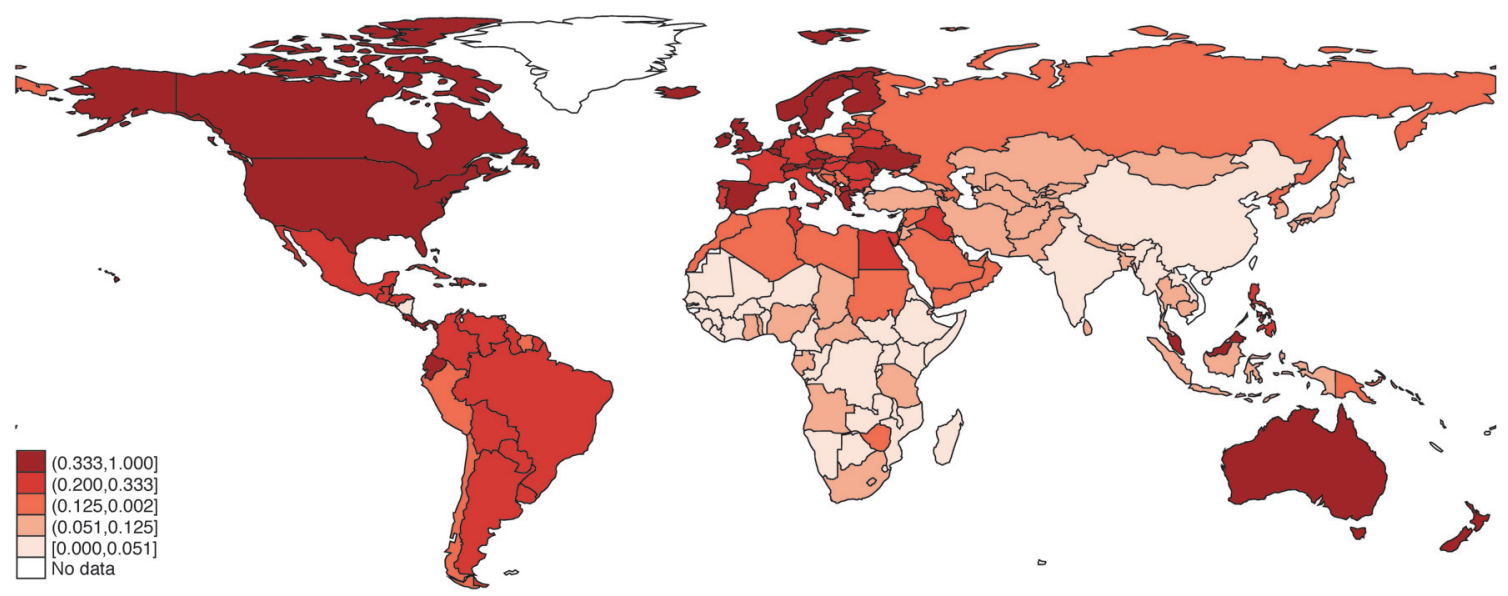

Abstract IDDF2020-ABS-0140 Figure 1 The global ratio of age-standardized incidence for oesophageal adenocarcinoma and squamous cell carcinoma 
incidence and risk factors of oesophageal cancer by histological subtypes using data from 178 countries.

Methods The data on the incidence of oesophageal cancer by histological types in 2018 were estimated from GLOBALCAN and Cancer Incidence in Five Continents (CI5). Age-standardized rates (ASRs) for oesophageal cancer incidence by histological subtypes were evaluated by Segi-Doll population. The prevalence of tobacco use, alcohol drinking, physical inactivity, obesity, diabetes, and lipid disorders for each country were retrieved from the Global Health Observatory. The association between the ratio of histological subtypes and risk factors was examined by multivariable linear regression.

Results We estimated a total of 63,470 (12.6\%) and 502,669 new cases of oesophageal adenocarcinoma (AC) and squamous cell carcinoma (SCC) in 2018, respectively. The incidence among males was 3.6-fold and 2.2-fold of that among females for AC and SCC, respectively. The highest AC:SCC ratio was found in the UK (ratio 2.880, ASR 7.5), New Zealand (2.667, 4.2), the Netherlands (2.536, 7.7), Bahrain (2.143, $0.9)$, and Canada $(2.000,3.7)$ among males (figure 1). As for females, the highest AC:SCC ratio was observed in Moldova $(1.000,0.2)$, the Netherlands $(0.800,1.2)$, Iceland $(0.750$; $0.5)$, the UK $(0.700 ; 1.4)$, and Cyprus $(0.667 ; 0.3)$. A higher AC:SCC ratio was associated with a higher prevalence of obesity (male: $\beta 0.039$, 95\% CI 0.023 to 0.055 ; female: 0.009 , 0.004 to 0.146 ) and high cholesterol (male: 0.028, 0.010 to 0.047; female: $0.011,0.004$ to 0.019 ); but a lower prevalence of tobacco use (male: $-0.007,-0.014$ to -0.001 ) and diabetes (male: $0.009,0.004$ to 0.146 ; female: $-0.021,-0.038$ to 0.003).

Conclusions While SCC is the predominant subtype of oesophageal cancer, the incidence of AC has surpassed SCC in a substantial proportion of countries, probably due to the increasing prevalence of obesity and metabolic disorders. Future research should investigate the reasons behind these epidemiological changes.

\section{IDDF2020-ABS-0141 THE GUT MICROBIOME AND SERUM METABOLOME ORCHESTRATE HEALTHY AGING AND LONGEVITY WITH NOVEL IMPLICATIONS FOR RENAL FUNCTION}

\begin{abstract}
${ }^{1}$ Zhiming Li*, ${ }^{2}$ Liang Sun, ${ }^{3}$ Caiyou Hu, ${ }^{1}$ Jiahong Ding, ${ }^{2}$ Qi Zhou, ${ }^{1}$ Yuzhe Sun, ${ }^{1}$ Rui Li, ${ }^{1}$ Hefu Zhen, ${ }^{4}$ Shuqin Sun, ${ }^{4}$ Jianmin Zhang, ${ }^{1}$ Chuanfa Liu, ${ }^{1}$ Mingyan Fang, 'Zhihuan Chen, ${ }^{3}$ Yuan Lv, ${ }^{4}$ Qizhi Cao, ${ }^{4}$ Yanan Sun, ${ }^{5}$ Zezhi Huang, ${ }^{2}$ Ruiyue Yang, ${ }^{2}$ Jun Dong, ${ }^{5}$ Ranhui Gong, ${ }^{5} J u n c h u n$ Li, ${ }^{1}$ Jie Ruan, ${ }^{1}$ Haorong Lu, ${ }^{3}$ Guofang Pang, ${ }^{3}$ Benjin He, ${ }^{3}$ Ninghu Li, ${ }^{1} \mathrm{TaO} \mathrm{Li}$, ${ }^{1}$ Wenbin Xue, ${ }^{1}$ Yan Li, ${ }^{1}$ Juan Shen, ${ }^{2}$ Fan Yang, ${ }^{2}$ Cheng Zhao, ${ }^{3}$ Qinghua Liang, ${ }^{1}$ Mingrong Zhang, ${ }^{2}$ Chen Chen, ${ }^{2}$ Huan Gong, ${ }^{2}$ Huiping Yuan, ${ }^{1}$ Yong Hou, ${ }^{1}$ Jian Wang, ${ }^{2}$ Ying Zhang, ${ }^{1}$ Huanming Yang, 'Shida Zhu, ${ }^{1}$ Jianping Cai, ${ }^{1}$ Liang Xiao, ${ }^{6}$ Haiyun Guo, ${ }^{6}$ Peng Zhao, ${ }^{2}$ Jian Li, ${ }^{1}$ Susanne Brix Pedersen, ${ }^{1}$ Xun $\mathrm{Xu}$, ${ }^{1} \mathrm{Huijue}$ Jia, ${ }^{1}$ Karsten Kristiansen, ${ }^{2}$ Ze Yang. 'BGl, China; ${ }^{2}$ The MOH Key Laboratory of Geriatrics, Beijing Hospital, National Center of Gerontology, China; ${ }^{3}$ Jiangbin Hospital, China; ${ }^{4}$ School of Gerontology, Binzhou Medical University, China; ${ }^{5}$ Office of Longevity Cultural, People's Government of Yongfu County, China; ${ }^{6}$ Department of International Exchange and Cooperation, Kunming Medical University, China
\end{abstract}

\subsection{6/gutjnl-2020-IDDF.87}

Background Aging is closely associated with the occurrence and development of many complex diseases such as cardiovascular conditions, type 2 diabetes, gastrointestinal (GI) and renal diseases. Detecting changes as early as possible in the human body due to aging is thus crucial significant to potentially mitigate its impacts on physical health.
Methods Here, we present a comprehensive metagenome association study and serum metabolomics profiling in a registry Guangxi-Longevity cohort aged from 20-111 years $(n=151)$ and Kunming cohort ages ranging from 20-80 years $(n=80)$.

Results We identified uremic toxins as key factors in serum metabolomics highly associated with aging, and this finding has been validated in an independent Kunming-Aging cohort aged from $20-80$ years $(n=80)$. We also observed that agingassociated systemic inflammation levels were positively associated with uremic toxins. Moreover, the increased Escherichia coli, Odoribacter splanchnicus, Bilophila wadsworthia and Parabacteroides spp. abundances were related to serum levels of uremic toxins, and the accumulating rate of uremic toxins and specific microbial species was robustly much slower in centenarians than in nonagenarians. We further investigated the frailty and health status in long-living individuals aged above 90 years, and found that the frailty status might be a putative extreme aging phenomenon characterized by novel uremic toxin accumulation patterns.

Conclusions Our findings reveal novel potential links between gut microbiota alterations, uremic toxins and aging, and highlight the preponderance of gut microbiota and serum metabolism in aging.

\section{IDDF2020-ABS-0142 PERFORMANCE INDICATORS OF ORGANISED COLORECTAL CANCER SCREENING PROGRAMMES USING FAECAL IMMUNOCHEMICAL TESTS AND COLONOSCOPY: A SYSTEMATIC REVIEW AND META-ANALYSIS}

${ }^{1}$ Hanyue Ding ${ }^{*},{ }^{1}$ Jiaye Lin, ${ }^{1}$ Zijun Xu, ${ }^{1}$ Xiao Chen, ${ }^{2}$ Harry Haoxiang Wang, ${ }^{1}$ Liwen Huang, ${ }^{1}$ Junjie Huang, ${ }^{3}$ Zhijie Zheng, ${ }^{1}$ Martin Chi-sang Wong. ${ }^{1} J C$ School of Public Health and Primary Care, Faculty of Medicine, Chinese University of Hong Kong, China; ${ }^{2}$ School of Public Health, Sun Yat-Sen University, China; ${ }^{3}$ School of Public Health, Peking University, China

\subsection{6/gutjnl-2020-IDDF.88}

Background The success of population-based colorectal cancer (CRC) screening is dependent on the optimal achievement of various targets. We estimated the pooled performance indicators of CRC screening programmes that used faecal immunochemical tests (FIT) as a primary screening modality and colonoscopy as a subsequent confirmatory test in various countries.

Methods We searched PubMed, Ovid MEDLINE, Embase, and Cochrane from inception to 1st Jan 2020. We included original articles published in the English language describing population-based CRC screening programme that used FIT and colonoscopy, and relevant national CRC screening reports by hand searching. We extracted data to pool early performance indicators, including participation rate, invalid FIT rate, FIT positive rate, adenoma/CRC detection rate of FIT, colonoscopy compliance rate, rate of adequate bowel preparation, colonoscopy completion rate, colonoscopy complication rate, and positive predictive values (PPV) of FIT for adenoma/CRC. We used Metaprop to conduct a meta-analysis via $\mathrm{R}$ software (version 3.6.3). The Freeman-Tukey double arcsine transformation was used to stabilise the variances, and a random-effects model was used to pool the rates with proportions.

Results A total of 85 studies (64 articles and 21 reports) were included in this meta-analysis. The pooled participation rate was 52.2\%, and the pooled proportion of invalid FIT was 